\section{Tratamiento quirúrgico con injerto óseo en fisuras alveolares de pacientes operados de labio y paladar fisurado: revisión de la literatura}

\author{
Surgical treatment with bone graft in \\ alveolar fissures of patients operated on \\ cleft lip and palate: review of the literature
}

\section{Resumen}

El labio y paladar fisurado (FLAP) se consideran como defectos anatómicos de profundo impacto estético como funcional lo cual lleva una serie de secuelas físicas, funcionales y psicológicas. Dentro del amplio tratamiento multidisciplinario que son sometidos los pacientes, el injerto alveolar secundario es fundamental para resolver diferentes secuelas como el defecto anatómico, la función y buscar continuar con el desarrollo adecuado del paciente. El objetivo del presente trabajo de investigación es hacer una revisión de la amplia literatura que existe entre los parámetros y consideraciones de esta técnica quirúrgica, ya que existe gran controversia entre los diversos estudios. El injerto alveolar secundario se realiza comúnmente entre los 9 y 12 años de edad durante la dentición mixta y antes de la erupción del canino permanente. Según la literatura el hueso esponjoso autólogo presenta los mejores resultados para este tipo de procedimientos, por sus diferentes mecanismos biológicos para la integración ósea. La zona dadora más utilizada sigue siendo la cresta ilíaca debido a su gran disponibilidad de tejido esponjoso y cortical, junto a su baja morbilidad. Actualmente los injertos óseos secundarios presentan una gran tasa de éxito y una reabsorción razonable, siendo el tratamiento más efectivo para las fisuras alveolares.

Palabras clave: Labio leporino; Regeneración ósea; Trasplante óseo (fuente: DeCS BIREME).

\footnotetext{
Abstract

The cleft lip and palate (FLAP) are considered as anatomical defects of deep aesthetic as well as functional impact which leads to a series of physical, functional and psychological sequelae. Within the extensive multidisciplinary treatment that patients undergo, the secondary alveolar graft is fundamental to resolve different sequelae such as anatomical defect, function and the search to continue with the proper development of the patient. The objective of this research is to review the extensive literature that exists between the parameters and considerations of this surgical technique, since there is great controversy among the various studies. The secondary alveolar graft is commonly performed between 9 and 12 years of age during mixed dentition and before the eruption of the permanent canine. According to the literature, autologous spongy bone presents the best results for
}

\section{Artículo de Revisión}

Carlos Campos Arenas ${ }^{1, a}$, Olinda Huapaya Paricoto ${ }^{1, b}$

${ }^{1}$ Universidad Nacional Mayor de San Marcos, Facultad de Odontología. Lima, Perú.

a Cirujano Dentista.

${ }^{\mathrm{b}}$ Especialista en Cirugía Oral y Maxilofacial.

\section{Correspondencia:}

Carlos Campos Arenas

Correo electrónico: ccarlos-ky@hotmail.com

Jr. Pazos \#140 Urb. Las Tejaditas, Barranco. Lima. Perú

Coautor:

Olinda Huapaya Paricoto

dhuapayap@unmsm.edu.pe

Editor:

Donald Ramos-Perfecto

Universidad Nacional Mayor de San Marcos, Perú.

Conflicto de intereses: los autores declaran no tener conflictos de interés.

Fuente de financiamiento: autofinanciado.

Recibido: 09/11/18

Aceptado: $28 / 03 / 19$

Publicado: 05/06/19

(C) Los autores. Este artículo es publicado por la revista Odontología Sanmarquina de la Facultad de Odontología, Universidad Nacional Mayor de San Marcos. Este es un artículo de acceso abierto, distribuido bajo los términos de la licencia Creative Commons Atribucion - No Comercia_Compartir Igual 4.0 Internacional. (http://creativecommons.org/licenses/by-nc-sa/4.0/) que permite el uso no comercial, distribución y reproducción en cualquier medio, siempre que la obra original sea debidamente citada. 
this type of procedure, due to its different biological mechanisms for bone integration. The most commonly used donor site continues to be the iliac crest due to its high availability of spongy and cortical tissue, together with its low morbidity. Nowadays, secondary bone grafts have a high success rate and a reasonable reabsorption, being the most effective treatment for alveolar fissures.

Keywords: Cleft lip; Bone regeneration; Bone transplantation (source: MeSH NLM).

\section{Introducción}

La labio y paladar fisurado (FLAP) es una de las malformaciones congénitas craneofaciales más frecuentes, la cual se produce por una alteración en la fusión de los tejidos que dan origen a labio superior y al paladar. Se considera como uno de los defectos anatómicos de gran impacto tanto estético como funcional que conlleva alteraciones futuras en el crecimiento y desarrollo, lo cual indica que se le debe brindar una atención médica integral por distintas especialidades de manera oportuna y eficaz ${ }^{1}$.

\section{Embriología}

La formación de la cara ocurre principalmente entre la cuarta y octava semana del desarrollo embrionario, experimentando cambios rápidos de forma y crecimiento, los dos primeros arcos son los responsables del desarrollo del rostro y cráneo. El desarrollo del rostro inicia a partir del ectomesénquima de la cresta neural que forman cinco primordios: la prominencia frontonasal, dos procesos maxilares y dos procesos mandibulares que rodean una depresión central. Durante la quinta y sexta semana los procesos maxilares derivados del primer arco braquial se fusionan con el proceso nasal medio para formar el labio superior, el alveolo y el paladar primario. El proceso nasal lateral forma las estructuras alares de la nariz, los procesos mandibulares forman el labio inferior y la mandíbula. El proceso de formación se da a partir de mecanismos celulares de proliferación, diferenciación, adherencia, apoptosis, desde el mesodermo se da la migración hacia la porción cefálica y la línea media invadiendo las uniones epiteliales entre los procesos, lo cual genera un engrosamiento de tejido mesenquimatoso, creando así una continuidad entre ambos procesos, lo que luego permitiría la formación de la estructura del labio superior y el piso nasal. La alteración de este fenómeno produce una carencia en la migración del tejido mesodérmico entre los procesos, lo cual finaliza con la reabsorción del puente de unión epitelial primitivo, alterando la fusión de los procesos y generando un surco profundo entre los procesos nasales mediales, laterales y el proceso maxilar ${ }^{2,3}$.

\section{Etiología}

Las fisuras labiales pueden ser unilaterales o bilaterales, y pueden afectar al alveolo o paladar, existen varios factores causales relacionados con la etiología, y a pesar de este número de factores contribuyentes reconocibles, no se conoce completamente su etiología, pero se concibe que su origen es multifactorial, compleja y permanente; según sus causas se pueden agrupar en dos grandes grupos ${ }^{4}$ :
Genéticas o intrínsecas. Actualmente se cree que del $40 \%$ de casos de LPF tiene una causa genética. Puede presentarse de manera única o asociada a síndromes como el síndrome de Down, de Patau, de Stickler, etc ${ }^{5}$.

Ambientales o extrínsecas. Existen agentes que alteran el desarrollo embriológico, llamados agentes teratógenos, entre los principales que están relacionados con el FLAP podemos mencionar:

- Agentes infecciosos. Infecciones como la rubéola congénita (togavirus) y la toxoplasmosis congénita ( $T o-$ xoplasma gondii) así también infecciones por citomagalovirus y sífilis congénita (Treponema pallidum) se han visto relacionadas como posibles causas ${ }^{6}$.

- Agentes farmacológicos. Existen efectos teratogénicos de ciertos fármacos asociados a esta patología, como los corticosteroides, benzodiacepinas, anticonvulsivantes, talidomina y antimetabolitos que antagonizan el metabolismo del ácido fólico. El consumo materno de alcohol y tabaco también se han mencionado como posibles agentes causales de esta patología ${ }^{7}$.

- Deficiencias metabólicas. Se encuentran la deficiencia de ácido fólico, diabetes gestacional, deficiencia de zinc y obesidad materna ${ }^{8}$.

\section{Epidemiología}

Las fisuras orofaciales, específicamente labio fisurado y paladar fisurado, son unos de los defectos de nacimiento más comunes. Ambos presentan epidemiología distinta, se estima de labio fisurado tiene una prevalencia estimada de 6,35 por cada 10000 nacidos vivos y para labio con paladar fisurado de 10,63 por cada 10000 nacidos vivos ${ }^{9}$. A nivel mundial se produce 1 en cada 500 - 2500 nacidos vivos, dependiendo de la ubicación geográfica, el sexo, la etnia y el estrato socioeconómico ${ }^{8}$.

Las principales diferencias de población han sido reportadas con tasas más altas en asiáticos y en nativos americanos ( 1 en 500 nacimientos) y en menor grado en los africanos ( 1 en 2500 nacimientos) ${ }^{10}$. Estudios en la región de las Américas, mostraron que la tasa global de labio paladar hendido es de 10,49 por cada 10000 nacidos vivos. Tasa que era superada por algunos países suramericanos; por ejemplo, Bolivia 23,7, seguida por Ecuador 14,96 y Paraguay con 13,3. Así mismo presentaron tasas más bajas países como Brasil con 10,12, Uruguay con 9,37, Perú con 8,94 , y Venezuela con 7,92 , todas ellas por cada 10000 nacidos vivos ${ }^{11}$. 
De acuerdo a las razas, se encuentra una incidencia más alta en la población China con 4,04 por cada 1000 nacimientos, seguida por la población nativa de América con 3,74 por cada 1000 nacimientos. Los afroamericanos tienen la más baja incidencia con 0,5 por cada 1000 nacimientos ${ }^{12}$.

\section{Clasificación de las fisuras}

Existen diferentes tipos de clasificaciones de las fisuras labio palatinas, debido a sus múltiples variantes patológicas y los diferentes criterios para establecer cada una de ellas. Una de las formas más aceptadas de nomenclatura y clasificación es con base a la descripción anatómica y estructuras involucradas. Mencionaremos solo esta clasificación por ser la que más se usa para el tratamiento quirúrgico ${ }^{13}$ :

Labio fisurado, con o sin paladar:

- Labio fisurado unilateral,

- Labio y paladar fisurado unilateral,

- Labio fisurado bilateral y

- Labio y paladar fisurado bilateral.

Paladar fisurado solo:

- Paladar fisurado,

- Paladar fisurado submucoso,

- Insuficiencia velofaríngea y

- Secuencia de Robin.

Cabe mencionar que a esta clasificación hace falta añadir las siguientes especificaciones:

- Incompleto: en el caso de que no sea total la extensión anatómica de la fisura labiopalatina.

- Primario: cuando compromete a la zona más anterior del paladar (premaxila).

- Secundario: cuando la región afectada es a partir del foramen oval.

\section{Tratamiento quirúrgico}

El tratamiento de las fisuras labio palatinas es sumamente complejo y tiene que realizarse por un equipo multidisciplinario que incluye cirujanos plásticos, otorrinolaringólogo, ortodoncista, cirujano maxilofacial, odontopediatra, fonoaudiólogos, psicólogos y el personal de enfermería. El tratamiento de estos pacientes debe iniciarse a muy temprana edad, debe hacerse el seguimiento correspondiente para cada etapa quirúrgica a través de los años hasta el completo desarrollo y maduración del esqueleto ${ }^{14}$.

En el 75\% de casos de labio y paladar fisurado, la fisura compromete la cresta alveolar, generando una fisura alveolar que imposibilita en muchas ocasiones el cierre de los tejidos blandos ${ }^{5}$. Habitualmente el tratamiento quirúrgico comprende dos etapas, la primera consiste en el cierre de los tejidos blandos del labio y paladar, y la segunda en la cual se hará el cierre óseo de la fisura alveolar y las correcciones de las deformidades dentofaciales para lo cual el paciente requiere ciertos requisitos como: ser mayor a los 7 años de edad y estar cursando con dentición mixta, tratamiento ortodontico y ortopédico para correcciones transversales, anteroposteriores y estabilización de los segmentos maxilares, tratamiento odontológico integral y evaluación de factores sistémicos de cada paciente ${ }^{13}$. Dentro del extenso tratamiento quirúrgico tomaremos únicamente el cierre de esta fisura alveolar, lo cual comúnmente es tratado mediante un injerto óseo autólogo, siendo ello parte fundamental en el tratamiento de fisuras alveolares uni y bilaterales ${ }^{15}$.

El objetivo principal de la reconstrucción de la hendidura maxilar es rellenar el hueso en el área de la fisura, lo que a su vez promueve el cierre de la fístula oronasal, establece la continuidad del arco maxilar, limita las alteraciones del crecimiento, brinda estabilidad entre los segmentos óseos y maximiza la estabilidad para las piezas permanentes. Ello hace posible la erupción de los dientes permanentes, dar simetría al arco, realzar la nariz, mejorar el habla, proporcionar tejido óseo para la futura colocación de implantes, mejorar la salud periodontal y facilitar la higiene bucal ${ }^{16-18}$.

\section{Injerto óseo alveolar}

El injerto alveolar secundario en pacientes con fisura alveolar fue sustentado por primera vez por Boyne y Sands ${ }^{19,20}(1972,1976)$. La operación se realiza bajo anestesia general, colgajos mucoperiósticos locales se levantan alrededor de la hendidura alveolar para definir completamente el defecto óseo; pasos importantes son la preparación cuidadosa del suelo nasal, la colocación del injerto óseo condesado en virutas de hueso esponjoso autógeno y un cierre hermético del tejido ${ }^{21}$.

La literatura no es concluyente con respecto al tiempo más favorable para el injerto óseo alveolar. Hay dos enfoques posibles con respecto al tiempo de la reconstrucción ósea alveolar: cirugía primaria de injerto óseo durante la infancia, y el injerto óseo secundario durante la etapa de dentición mixta ${ }^{22}$.

Injerto alveolar primario. El injerto alveolar primario implica la reconstrucción alveolar en asociación con la reparación de los tejidos blandos del labio durante la infancia. El objetivo de la reparación de la hendidura alveolar realizada antes del cierre del paladar es prevenir los efectos limitantes del cierre quirúrgico del paladar en el desarrollo temprano del tercio medio de la cara. No todos los pacientes son adecuados para el injerto primario, deben cumplir los siguientes criterios: la fisura palatina debe ser incluyendo el paladar duro y los segmentos alveolares deben estar correctamente alineados ortodónticamente antes del injerto. Se han observado alteraciones y deficiencias en el crecimiento y desarrollo hemifacial en algunos de estos pacientes sometidos a este injerto primario ${ }^{23}$.

Injertos óseos secundarios. El injerto alveolar secundario es el más atractivo y el método más popular para 
tratar las fisuras alveolares. Se suele realizar cuando la mitad de la raíz del canino permanente del lado de la fisura está casi completa, en este punto se muestra acelerado y activo para la erupción, además que la mayor parte del crecimiento y desarrollo del rostro esta completado. Esto corresponde a la edad de 9 a 12 años ${ }^{24}$.

El principal objetivo de la reconstrucción secundaria del hueso alveolar es: proporcionar una matriz ósea madura que soporta el movimiento canino y la formación del arco dental estable, facilitar el movimiento ortodóntico de los dientes, la rehabilitación y reconstrucción protésica, el cierre de la fistula oro nasal, y proporcionar soporte óseo para el labio y la nariz ${ }^{25}$.

\section{Mecanismos de cicatrización del injerto óseo y formación ósea}

Los diversos mecanismos de formación ósea dependen de los orígenes y composiciones del injerto que tienen un potencial de regeneración ósea diferente asociado a las siguientes propiedades ${ }^{26}$ :

- Osteogénesis: los osteoblastos vivos derivados del injerto contribuyen a la producción de hueso nuevo.

- Osteoinducción: estimulación de células osteoprogenitoras que se diferencian en osteoblastos, generalmente influenciadas por una proteína morfogenética ósea (BMP) liberada del injerto.

- Osteoconducción: los injertos proporcionan un "esqueleto" que ayuda a los capilares y las células óseas precursoras a desarrollarse, creando así un andamio donde se puede crear hueso alrededor de él.

El material ideal para la rehabilitación ósea debe tener las siguientes características ${ }^{27}$ :

- Osteogénico, propiedades de osteoinducción y osteoconducción,

- Estimulación de la neoangiogénesis,

- Falta de reacciones antigénicas, teratogénicas o carcinogénicas,

- Suministro en cantidades suficientes,

- Apoyo satisfactorio y estabilidad,

- Morbilidad mínima a cero complicaciones,

- Naturaleza hidrófila,

- Fácil de manejar y

- Bajo costo.

La integración del injerto en el sitio receptor es un procedimiento que incluye varias etapas: inflamación, revascularización, osteoinducción, osteoconducción y finalmente remodelación. Para lograr regeneración ósea, los injertos deben exhibir tres elementos fundamentales ${ }^{28}$ :

- Osteoprogenitor de células mesenquimales u osteoblastos vivos,
- Factores de crecimiento que son beneficiosos para el proceso regenerativo $y$

- Un "esqueleto" con la capacidad mecánica de apoyo para la adhesión de las células, lo que conduce aún más a su crecimiento y proliferación.

La histología de los injertos corticales y esponjosos desempeñan un papel significativo en su comportamiento biológico que puede resumirse de la siguiente forma ${ }^{29}$ :

- Los injertos esponjosos estimulan la osteogénesis debido a la presencia de osteoblastos, osteocitos y células madre mesenquimales dentro de su estructura.

- Los injertos corticales proporcionan principalmente estabilidad, sin embargo, tienen capacidad osteogénica deficiente, exhiben una reabsorción prolongada, mientras que el crecimiento del hueso nuevo es lento.

- Una combinación de injertos corticales y esponjosos puede garantizar la estabilidad y la osteogénesis.

Existen también diferencias en la resistencia mecánica que aumenta en los huesos esponjosos debido a la tasa de deposición más rápida del tejido óseo nuevo mientras que en injertos corticales la resistencia disminuye en un $40 \%$ desde las primeras 6 semanas hasta los 6 meses posteriores a la cirugía ${ }^{30}$.

\section{Clasificación de los injertos óseos}

Según la naturaleza del injerto lo podemos clasificar en:

Autólogos. El material óseo para el injerto es obtenido del mismo paciente. No hay propiedades antigénicas ya que el donante y receptor son la misma persona. Este injerto es el único que cumple con las tres vías para la formación de hueso (osteogénesis, osteinducción y osteoconducción), llegan a tener una taza de éxito > 95\%. La principal desventaja es la creación de un segundo trauma que a veces puede afectar la salud sistémica del paciente especialmente en los casos de extenso volumen óseo ${ }^{31}$.

Aloinjertos. El material óseo es tomado de otros individuos de la misma especie, pero de diferentes genotipos, se obtienen de cadáveres se almacenan y procesan en bancos. Tiene la ventaja de que evita la intervención quirúrgica en el sitio donante. Estos pueden formar hueso a través de la osteoinducción y la osteoconducción solamente. No forma osteogénesis ya que no posee células vivas, por lo tanto, la formación ósea es lenta y pierde un volumen apreciable con respecto a los injertos autólogos. Son procesados para neutralizar la respuesta inmune y la transmisión de enfermedades infecciosas. Están disponibles como cortical, esponjoso o cortico-esponjoso ${ }^{32}$.

Xenoinjertos. Son obtenidos por individuos de diferentes especies para el receptor, por lo general poseen características osteoconductoras con potencial de reabsorción limitado y son combinadas con factores de crecimiento o injertos óseos de otro origen. Entre sus principales desventajas están el hecho de que las características óseas 
difieren en comparación a los humanos, además que su procesamiento podría afectar sus propiedades físico-químicas como en los aloinjertos ${ }^{33}$.

Materiales aloplásticos. Sustituto desarrollados para imitar el tejido óseo natural. Cubren una amplia gama de sustitutos óseos o aplicaciones de soporte de tejidos blandos, disponibles en muchos tamaños y formas, se han empleado varias técnicas incluyendo la superficie, textura, formación de capas mineralizadas y el uso de biorreactores para que el producto final sea capaz de imitar el ambiente en el que crecen los osteoblastos naturalmente. Estos materiales biomiméticos se caracterizan por ser osteoconductores sin capacidad osteoinductiva o potencial osteogénico celular. Funciona como un andamio para apoyar el crecimiento celular y la formación de hueso, aumentar la adhesión y proliferación celular ${ }^{34}$.

Se considera el defecto del proceso alveolar de los pacientes con labio y paladar fisurado como un defecto marginal de la continuidad en la abertura piriforme y el alveolo. Así la reconstrucción de la hendidura alveolar se supone que es un injerto inlay entre ambos segmentos óseo, en lugar de un injerto onlay en el maxilar ${ }^{35}$. Actualmente siguen siendo los injertos autólogos la mejor opción, entre los injertos óseos, para el relleno de la fisura alveolar. Los principales sitios de donación autóloga para la reconstrucción ósea de la región maxilofacial, por sus características histológicas y de osificación, así como el sitio del abordaje y la cantidad de hueso a obtener, son:

- Cresta Iliaca: la cresta ilíaca posterior se considera como la fuente óptima de hueso autógeno para la reconstrucción de la hendidura alveolar, actualmente se le denomina "gold standard injerto óseo". La cresta iliaca anterior tiene la ventaja de proporcionar una gran cantidad de hueso esponjoso y fácil acceso quirúrgico. Además, tiene un gran potencial osteogénico especialmente en la fase temprana después del injerto, debido a su abundante capacidad pluripotente de las células precursoras osteogénicas. El hueso esponjoso es considerado superior al cortical porque es relativamente fácil retirarlo, reduce el tiempo operatorio y contiene una mayor cantidad de células osteogénicas. Además, el hueso esponjoso colocado en la fisura alveolar es predecible y tiene una mayor tasa de éxito. Sin embargo, los colgajos musculo - periósticos durante la exposición quirúrgica de la cresta iliaca puede dar lugar a morbilidades post operatorias significativas incluyendo hematoma, dolor, malestar, dificultad para caminar y una hospitalización prolongada, aunque estas son poco frecuentes $28,35,36$.

- Calota craneal: el hueso de calota craneal se obtiene mediante una intervención que tiene baja morbilidad y un éxito cercano al de la cresta iliaca, por lo cual se debería considerar con mayor frecuencia como una alternativa viable para los pacientes con FLAP ${ }^{37}$. Entre las ventajas de la calota craneal se destaca la ausencia de dolores post operatorios, en comparación la cresta iliaca deja una cicatriz poco o nada visible, menor reabsorción del hueso membranoso con el hueso endocondral. La principal desventaja es la escasa maleabilidad debido a su gran componente cortical. Presenta, en muy bajo porcentaje, complicaciones como hematoma epidural, fístula del líquido cefalorraquídeo y daño cerebral ${ }^{38}$.

- Costilla: fue el primer sitio donante elegido para este tipo de injertos, sin embargo, estudios actuales refieren que presentan más del 50\% de reabsorción del injerto, complicaciones como dehiscencia de la herida y reaparición de la fistula oro - nasal. Claramente, el injerto de costilla no ofrece ventajas suficientes en comparación con el injerto de cresta iliaca ${ }^{17}$.

- Región mentoniana o sínfisis mandibular: los injertos mandibulares parecen ser muy eficaces en la formación de puentes óseos y en permitir la erupción de los dientes permanentes. Presenta menos complicaciones y reabsorción que un injerto de costilla. Sin embargo, debido a lo reducido de zona donante muchas veces no es posible cerrar los defectos amplios que presentan las fisuras alveolares ${ }^{39}$.

\section{Técnica quirúrgica}

La técnica quirúrgica es una modificación de la descrita por Boyne y Sands ${ }^{21}$ debe ser realizada por todo un equipo quirúrgico, uno encargado del lecho receptor y otro encargado de la zona dadora. Debe ser un equipo capacitado y con vasta experiencia en este tipo de tratamiento. El procedimiento se realiza mediante anestesia general con intubación nasoendotraqueal.

La técnica se describe a continuación paso por paso ${ }^{40}$ : se realiza a nivel de mucosa una incisión desde la hendidura hasta distal del primer molar a nivel del margen gingival con una liberante en distal de 45 grados de 1 cm (Figura 1).

El patrón de incisión fue a espesor total decolado bilateralmente. Se realiza la disección de los planos con sumo cuidado y por planos, nasal, palatino y alveolar, evidenciando la fistula. (Figura 2).

La mucosa nasal y palatina son suturadas firmemente, se reconstruye la base mucosa del piso nasal, cerrando así la fistula. El suelo nasal es reconstruido con hueso cortical iliaco (Figura 3).

Incisión de liberación perióstica para asegurar la mejor movilidad del colgajo, se realiza la transposición de los colgajos para el cierre sin tensión. La cicatrización de la región distal de la molar será por segunda intención (Figura 4).

\section{Conclusiones}

El injerto óseo secundario se presenta como la mejor alternativa para el tratamiento de las fisuras naso alveolares, su tasa de éxito es variable según los autores 


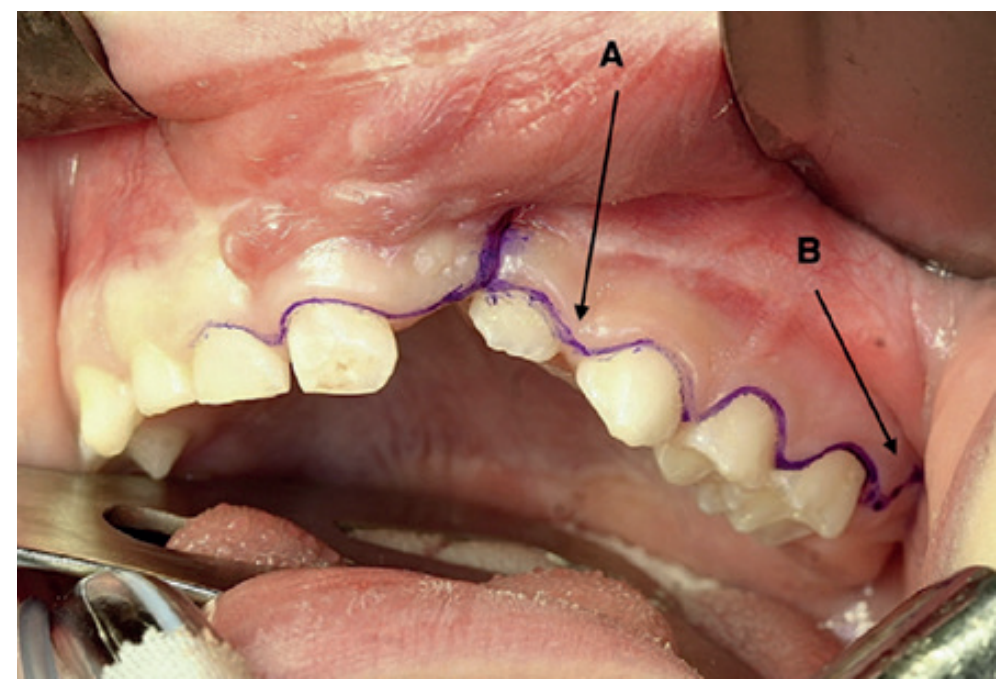

Figura 1. Diseño de aleta gingival. A. Incisión surcular con una incisión vertical en la hendidura de la región. B. Incisión de la hendidura con angulación distal de $45^{\circ}$. Fuente: Lopez et al. ${ }^{40}$

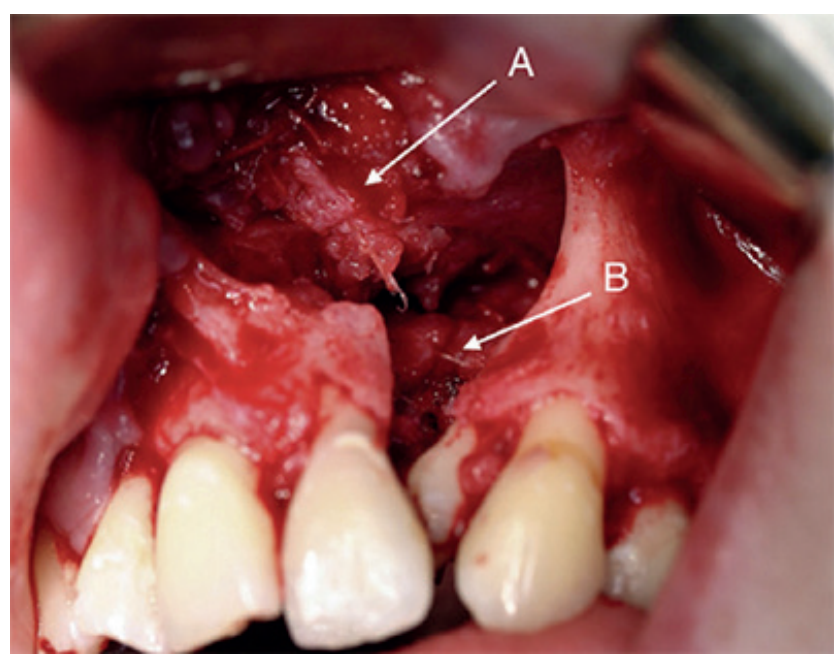

Figura 2. Cierre de mucosa. A. Mucosa nasal. B. Mucosa palatina. Fuente: Lopez et al. ${ }^{40}$

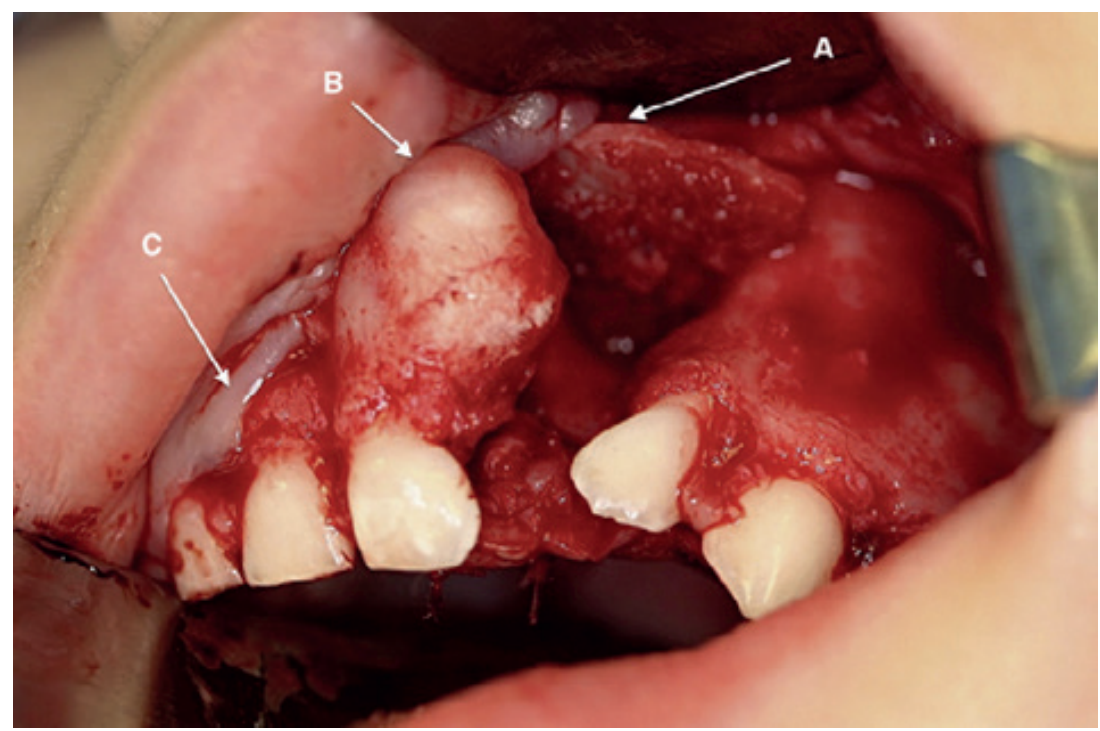

Figura 3. Disección y reconstrucción de la fisura alveolar. A. Cortical iliaca colocada entre el plano de mucosa nasal y el hueso esponjoso particulado que será agregado. B. Premaxila. C. Colgajo de aleta, elevado a espesor total. Fuente: Lopez et al. ${ }^{40}$ 


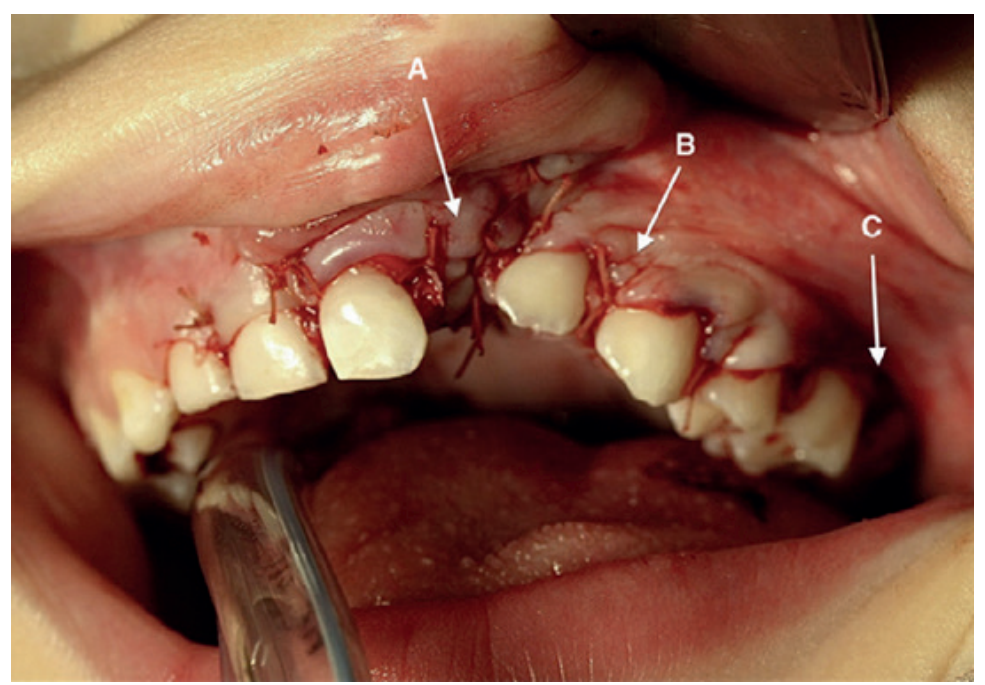

Figura 4. Cierre de las aletas gingivales. A. Cierre sobre la hendidura sin tensión. B. Transposición papilar gingival. C. Área de curación secundaria. Fuente: Lopez et al. ${ }^{40}$

3,5,13,15,20,21,29,37, y presenta una baja morbilidad si es empleado de la manera adecuada. Proporciona una solución estable como cubrir el defecto óseo, cerrar la fistula oronasal, lograr estabilidad de los segmentos maxilares, permitir la erupción del canino permanente, proporciona tejido óseo para la futura rehabilitación, mejora la proyección del ala nasal y la estética facial.

El injerto óseo autólogo de tipo mixto sigue siendo el que mejores resultados ha presentado debido a sus mecanismos biológicos de integración ${ }^{15,18,25,29}$. De las zonas donantes para el injerto óseo, la cresta iliaca es el más empleado, debido a gran volumen de hueso mixto, la baja morbilidad y la razonable reabsorción del injerto ${ }^{25}$. Aunque la bibliografía no es concluyente sobre la edad óptima para la realización de esta cirugía, se viene realizando con frecuencia y éxito, el injerto alveolar secundario durante la dentición mixta, entre los 9 y 12 años de edad, teniendo en vital consideración el grado de erupción del canino permanente ${ }^{3,15}$. La técnica quirúrgica debe ser pulcra e individualizada, debe presentar un colgajo amplio, reconstruir el piso nasal y el plano palatino para el correcto cierre de la fístula, generar campo para la colocación del injerto óseo de manera uniforme y tener un cierre sin tensión a lo largo de la hendidura.

Se hace necesario realizar trabajos de investigación a largo plazo, así como explorar nuevas formas de aumentar la integración del injerto óseo, reducir la morbilidad, las complicaciones y el tiempo operatorio, de manera que pueda afianzarse aún más su empleo como protocolo del tratamiento de las fisuras alveolares y así mejorar la calidad de vida de este grupo de pacientes.

\section{Referencias bibliográficas}

1. Becker DB, Coalson RS, Sachanandani NS, Fair D, Lugar HM, Kirchner LE, et al. Functional neuroanatomy of lexical processing in children with cleft lip and palate. Plast Reconstr Surg. 2008;122(5):1371-81.
2. López AM, Rodríguez HR, Solís CL. Nasoalveolar bone graft integration range in patients. Rev Odontológica Mex. 2012;16(1):18-30.

3. Chigurupati R. Cleft Lip and Palate: Timing and Approaches to Reconstruction. In: Current Therapy in Oral and Maxillofacial Surgery. 1ra Edición. Missouri. Elsevier; 2012. p. 726-50.

4. Marazita ML, Mooney MP. Current concepts in the embryology and genetics of cleft lip and cleft palate. Clin plast Surg. 2004;31(2):125-40.

5. Kazemi A, Stearns JW, Fonseca RJ. Secondary grafting in the alveolar cleft patient. Oral Maxillofac Surg Clin North Am. 2002;14(4):477-90.

6. Wyszynski DF, Beaty TH. Review of the role of potential teratogens in the origin of human non-syndromic oral clefts. Teratology 1996; 53:309-17.

7. Cohen MM. Etiology and pathogenesis of orofacial clefting. Oral Maxillofac Surg Clin North Am. 2000;3:361376.

8. Shkoukani MA, Lawrence LA, Liebertz DJ, Svider PF. Cleft palate: A clinical review. Birth Defects Res Part C Embryo Today Rev. 2014;102(4):333-42.

9. Parker SE, Mai CT, Canfield MA, et al. Updated National Birth Prevalence estimates for selec birth defects in the United States, 2004 - 2006. Birth Defects Res. 2010;88:1008-16.

10. Parada C, Chai Y. Roles of BMP Signaling Pathway in Lip and Palate Development. Front Oral Biol. 2013;16:60-70.

11. Poletta FA, Castilla EE, Regional analysis on the occurrence of oral clefts in South America. Am J Med Genet A. $2007 ; 143(24): 3216-27$.

12. Vanderas AP. Incidence of cleft lip, cleft palate, and cleft lip and palate among races: a review. Cleft Palate J 1987; 24:216-25.

13. Montaño A, Rincón H, Landa C. Grado de integración de injertos óseos nasoalveolares, en pacientes con 
secuelas de labio y paladar fisurados. Rev Odontol Mex. 2012;16(1):18-30.

14. Ford M, Tastets H. Caceres R. Tratamiento de la fisura palatina. Rev Med Clin Condes. 2010;21(1):16-25.

15. Guo J, Li C, Zhang Q, Wu G, Deacon S, Chen J, et al. Secondary bone grafting for alveolar cleft in children with cleft lip or cleft lip and palate. Cochrane Database Syst Rev. 2011;15(6):1 -25.

16. Bajaj A, Wongworawat A, Punjabi A. Management of alveolar clefts. J Craniofac Surg. 2003;14(6):840-6.

17. Meyer S, Mølsted K. Long-term outcome of secondary alveolar bone grafting in cleft lip and palate patients: a 10-year follow-up cohort study. J Plast Surg Hand Surg. 2013;47(6):503-8

18. Miloro M, Ghali GE, Larse P, Waite P. Peterson's Principles of Oral and Maxillofacial Surgery. 2a ed. London: BC Decker editores; 2004.

19. Boyne PJ, Sands NR. Combined orthodontic surgical management of residual palate-alveolar defect. J Oral Maxillofac Surg. 1976;70(1):20-37.

20. Boyne PJ, Sands NR. Secondary bone grafting of residual alveolar and palatal defect. J Oral Maxillofac Surg. 1972;30(2):87-92.

21. Paterson M, Rae J, Paterson P, Gilgrass T, Devlin M, McIntyre G. Secondary alveolar bone grafting (CLEFTSiS) 2007-2010. Cleft Palate-Craniofacial J. 2016;53(2):141-6.

22. Eichhorn W, Blessmann M, Pohlenz P, Blake FA, Gehrke G, Schmelzle R, Heilamd M. Primary osteoplasty using calvarian bone in patients with cleft lip, alveous and palate. J Cranio maxillofac. Surg. 2001; 38:155-63.

23. Rosenstein SW. Early bone grafting of alveolar cleft deformities.J. Oral Maxillofac. Surg. 2003;61(9):1078-1081.

24. Hynes PJ, Earley MJ. Assessment of secondary alveolar bone grafting using a modification of the Bergland grading system. Br. J. Plastic. Surg. 2003;56(7):630-36.

25. Baqain ZH, Anabtawi M, Karaky AA, Malkawi Z. Morbidity from anterior iliac crest bone harvesting for secondary alveolar bone grafting: an outcome assessment study. J. Oral Maxillofac. Surg. 2009;67(3):570-75.

26. Bauer TW, Muschler GF. Bone graft materials. An overview of the basic science. Clin Orthop Relat Res. 2000;371:10-27.

27. Janicki P, Schmidmaier G. What should be the characteristics of the ideal bonegraft substitute? Combining scaffolds with growth factors and/or stem cells. Injury. 2011;42(2):77-81.

28. Roberts WE, Simmons KE, Garetto LP, DeCastro RA. Bone physiology andmetabolism in dental implantology: risk factors for osteoporosis and othermetabolic bone diseases. Implant Dent 1992;1:11-21.

29. Titsinides S, Agrogiannis G, Karatzas T. Bone grafting materials in dentoalveolar reconstruction: A comprehensive review. Jpn Dent Sci Rev. 2019;55(1):26-32.

30. Ronden DJ. Principles of Bone Grafting. Oral Maxillofac Surg Clin NA. 2010;22(3):295-300.

31. Sakkas A, Wilde F, Heufelder M, Winter K, Schramm A. Autogenous bone grafts in oral implantology - is it still a "gold standard"? A consecutive review of 279 patients with 456 clinical procedures. Int J Implant Dent. 2017;3:23. DOI 10.1186/s40729-017-0084-4

32. Delloye C, Cornu O, Druez V, Barbier O. Bone allografts: what they can offer and what they cannot. J Bone Jt Surg. 2007;89(5):574-9.

33. Tovar N, Jimbo R, Gangolli R, Perez L, Manne L, Yoo D, et al. Evaluation of bone response to various anorganic bovine bone xenografts: an experimental calvaria defect study. Int J Oral Maxillofac Surg. 2014;43(2):251-60.

34. AlGhamdi AS, Shibly O, Ciancio SG. Osseous grafting part II: xenografts and allo-plasts for periodontal regeneration - a literature review. J Int Acad Periodontol. 2010;12(2):39-44.

35. Rawashdeh MA, Telfah H. Secondary alveolar bone grafting: the dilemma of donor site selection and morbidity. Br. J. Oral Maxillofac. Surg. 2008;46(8):665-670.

36. Swan MC, Goodacre TEE. Morbidity at the iliac crest donor site following bone graffing of the cleft alveolus. Br. J Oral Maxillofac. Surg. 2006;44(2):129-33.

37. Hudak K, Hetinger P, Denny A. Cranial bone grafting for alveolar clefts: a 25 year review of outcome. Plast Reconstr Surg. 2014;133(5):662-8.

38. Donovan MG, Dickerson NC, Hanson LJ, Gustafson RB. Maxillary and mandibular reconstruction using calvarial bone grafts and Branemark implants: A preliminary report. J Oral Maxillofac Surg 1994;52:588-94.

39. Mikoya T, Inoue N, Matsuzawa Y, Totsuka Y, Kajii T, Hirosawa T. Monocortical strategies for alveolar cleft reconstruction: a systematic review of the literature. Clin Oral Investig. 2014;18(1):219-26.

40. Lopez C, Gonzales L, Alvaro F. Isolated keratinized gingiva incisión in alveolar cleft bone grafts improves qualitative outcomes: A single surgeon's 23 year experience. Journal of Cranio-Maxillo-Facial Surgery. 2014;42(8):1692-97. 